Moroccan J. of Pure and Appl. Anal. (MJPAA)

Volume 7(1), 2021, Pages 30-42

ISSN: Online 2351-8227 - Print 2605-6364

DOI: $10.2478 / \mathrm{mjpaa}-2021-0004$

\title{
Subordination results for a class of analytic functions
}

\section{K. Aouf ${ }^{1}$, B. A. Frasin ${ }^{2}$ and G. Murugusundaramoorthy ${ }^{3}$}

AвSTRACT. In this paper, we derive several subordination results and integral means result for certain class of analytic functions with complex order defined by means of q-differential operator. Some interesting corollaries and consequences of our results are also considered

Mathematics Subject Classification (2020). 30C45, 30C50, 05A30.

Key words and phrases. Analytic functions, Univalent functions, Subordinating factor sequence, $q$-difference operator, Hadamard product (or convolution).

\section{Introduction and definitions}

Let $\mathcal{A}$ be the class of functions which are analytic in the open unit disc $\mathbb{U}=\{z \in \mathbb{C}:|z|<1\}$ of the form

$$
f(z)=z+\sum_{n=2}^{\infty} a_{n} z^{n}
$$

Received : 19 August 2020 - Accepted: 26 October 2020.

(C)The Author(s) 2020. This article is published with open access by Sidi Mohamed Ben Abdallah University.

${ }^{1}$ Department of Mathematics, Faculty of Science, Mansoura University, Mansoura 35516, Egypt.

e-mail:mkaouf127@yahoo.com

${ }^{2}$ Faculty of Science, Department of Mathematics, Al al-Bayt University, Jordan

e-mail: bafrasin@yahoo.com (Corresponding Author).

${ }^{3}$ Department of Mathematics,School of Advanced Sciences, Vellore Institute of Technology (Deemed to be University), Vellore - 632014, India.

e-mail: gmsmoorthy@yahoo.com . 
We denote by $\mathcal{S}$ the subclass of $\mathcal{A}$ consisting of functions which are analytic, univalent in $\mathbb{U}$ and normalized by $f(0)=0=f^{\prime}(0)-1$. The well known subclasses of $\mathcal{S}$ are the class of starlike functions $\mathcal{S}^{*}$ and convex functions $\mathcal{K}$. For given $g(z)=z+\sum_{n=2}^{\infty} b_{n} z^{n} \in \mathcal{S}$, the Hadamard product of $f$ and $g$ is defined by

$$
(f * g)(z)=z+\sum_{n=2}^{\infty} a_{n} b_{n} z^{n}=(g * f)(z) .
$$

We note that $f * g \in \mathcal{S}$ is analytic and univalent in the open disc $\mathbb{U}$.

For two analytic functions $f, g \in \mathcal{A}$ we say that $f$ is subordinate to $g$, denoted by $f \prec g$, if there exists a Schwarz function $\omega(z)$ which is analytic in $\mathbb{U}$ with $\omega(0)=0$ and $|\omega(z)|<1$ for all $z \in \mathbb{U}$, such that $f(z)=g(\omega(z))$ for $z \in \mathbb{U}$. Note that, if the function $g$ is univalent in $\mathbb{U}$, due to Miller and Mocanu [28](see[18]), we have

$$
f(z) \prec g(z) \Longleftrightarrow f(0)=g(0) \text { and } f(\mathbb{U}) \subset g(\mathbb{U}) .
$$

Now we recall here the notion of $q$-operator i.e. q-difference operator that play vital role in the theory of hypergeometric series, quantum physics and in the operator theory. The application of $q$-calculus was initiated by Jackson [23], recently Kanas and Răducanu [24] (also see [1, 2, $16,21,22,23,33,34])$ have used the fractional q-calculus operators in investigations of certain classes of functions which are analytic in $\mathbb{U}$.

Let $0<q<1$. For any non-negative integer $n$, the $q$-integer number $n$ is defined by

$$
[n]_{q}=\frac{1-q^{n}}{1-q}=1+q+\cdots+q^{n-1}, \quad[0]_{q}=0 .
$$

In general, we will denote

$$
[x]_{q}=\frac{1-q^{x}}{1-q}
$$

for a non-integer number $x$. Also the $q$-number shifted factorial is defined by

$$
[n]_{q} !=[n]_{q}[n-1]_{q} \ldots[2]_{q}[1]_{q}, \quad[0]_{q} !=1 .
$$

Clearly,

$$
\lim _{q \rightarrow 1^{-}}[n]_{q}=n \quad \text { and } \quad \lim _{q \rightarrow 1^{-}}[n]_{q} !=n ! .
$$

For $0<q<1$, the Jackson's $q$-derivative operator (or $q$-difference operator) of a function $f \in \mathcal{A}$ given by (1.1) defined as follows [23]:

$$
\mathfrak{D}_{q} f(z)=\left\{\begin{array}{cc}
\frac{f(z)-f(q z)}{(1-q) z} & \text { for } z \neq 0 \\
f^{\prime}(0) & \text { for } z=0
\end{array},\right.
$$

$\mathfrak{D}_{q}^{0} f(z)=f(z)$, and $\mathfrak{D}_{q}^{m} f(z)=\mathfrak{D}_{q}\left(\mathfrak{D}_{q}^{m-1} f(z)\right), m \in \mathbb{N}=\{1,2, \ldots\}$. From (1.5), we have

$$
\mathfrak{D}_{q} f(z)=1+\sum_{n=2}^{\infty}[n]_{q} a_{n} z^{n-1} \quad(z \in \mathbb{U}),
$$


where $[n]_{q}$ is given by (1.3). For a function $\psi(z)=z^{n}$, we obtain

$$
\mathfrak{D}_{q} \psi(z)=\mathfrak{D}_{q} z^{n}=\frac{1-q^{n}}{1-q} z^{n-1}=[n]_{q} z^{n-1}
$$

and

$$
\lim _{q \rightarrow 1^{-}} \mathfrak{D}_{q} \psi(z)=\lim _{q \rightarrow 1^{-}}\left([n]_{q} z^{n-1}\right)=n z^{n-1}=\psi^{\prime}(z),
$$

where $\psi^{\prime}$ is the ordinary derivative.

Let $t \in \mathbb{R}$ and $n \in \mathbb{N}$. The q-generalized Pochhammer symbol is defined by

$$
[t ; n]_{q}=[t]_{q}[t+1]_{q}[t+2]_{q} \ldots[t+n-1]_{q}
$$

and for $t>0$ the q-gamma function is defined by

$$
\Gamma_{q}(t+1)=[t]_{q} \Gamma_{q}(t) \quad \text { and } \quad \Gamma_{q}(1)=1 .
$$

Using the $q$-difference operator, Kanas and Raducanu [24] defined the Ruscheweyh $q$-differential operator as below: For $f \in \mathcal{A}$,

$$
\mathcal{R}_{q}^{\delta} f(z)=f(z) * F_{q, \delta+1}(z) \quad(\delta>-1, z \in \mathbb{U})
$$

where

$$
F_{q, \delta+1}(z)=z+\sum_{n=2}^{\infty} \frac{\Gamma_{q}(n+\delta)}{[n-1]_{q} ! \Gamma_{q}(1+\delta)} z^{n}=z+\sum_{n=2}^{\infty} \frac{[\delta+1 ; n]_{q}}{[n-1]_{q} !} z^{n}
$$

Making use of (1.9) and (1.10), Aldweby and Darus[1] defined the $q$-analogue of Ruschewey operator $\mathcal{R}_{q}^{\delta}: \mathcal{A} \rightarrow \mathcal{A}$ as follows:

$$
\begin{aligned}
\mathcal{R}_{q}^{\delta} f(z) & =z+\sum_{n=2}^{\infty} \frac{\Gamma_{q}(n+\delta)}{[n-1]_{q} ! \Gamma_{q}(1+\delta)} a_{n} z^{n} \quad(z \in \mathbb{U}) . \\
& =z+\sum_{n=2}^{\infty} \Theta_{n}(q, \delta) a_{n} z^{n} \quad(z \in \mathbb{U}) .
\end{aligned}
$$

where

$$
\Theta_{n}:=\Theta_{n}(q, \delta)=\frac{\Gamma_{q}(n+\delta)}{[n-1]_{q} ! \Gamma_{q}(1+\delta)}
$$

As $q \rightarrow 1^{-}$,we note that

$$
\begin{aligned}
& \mathcal{R}_{q}^{0} f(z)=f(z), \\
& \mathcal{R}_{q}^{1} f(z)=z \mathfrak{D}_{q} f(z)=z f^{\prime}(z)
\end{aligned}
$$

It is easy to check that

$$
z \mathfrak{D}_{q}\left(F_{q, \delta+1}(z)\right)=\left(1+\frac{[\delta]_{q}}{q^{\delta}}\right) F_{q, \delta+2}(z)-\frac{[\delta]_{q}}{q^{\delta}} F_{q, \delta+1}(z) \quad(z \in \mathbb{U}) .
$$


Making use of (1.9), (1.13) and the properties of Hadamard product, we obtain the following equality( see [24])

$$
\left.\left.z \mathfrak{D}_{q}\left(\mathcal{R}_{q}^{\delta} f(z)\right)=\left(1+\frac{[\delta]_{q}}{q^{\delta}}\right) \mathcal{R}_{q}^{\delta+1} f(z)\right)-\frac{[\delta]_{q}}{q^{\delta}} \mathcal{R}_{q}^{\delta} f(z)\right) \quad(z \in \mathbb{U}) .
$$

From (1.11), we note that

$$
\lim _{q \rightarrow 1^{-}} F_{q, \delta+1}(z)=\frac{z}{(1-z)^{\delta+1}}, \quad \lim _{q \rightarrow 1^{-}} \mathcal{R}_{q}^{\delta} f(z)=f(z) * \frac{z}{(1-z)^{\delta+1}} .
$$

Thus, when $q \rightarrow 1^{-}$we can say that Ruscheweyh $q$-differential operator reduces to the differential operator defined by Ruscheweyh [32] (see[6, 9, 10, 11, 12]) and (1.14) gives the well known recurrent formula for Ruscheweyh differential operator. With the help of the differ-

ential operator $\mathcal{R}_{q}^{\delta}$, given by (1.11) we say that a function $f \in \mathcal{A}$ is said to be in the class $\mathcal{F}_{\lambda, q}(b, M)$ if it satisfies

$$
\left|\frac{b-1+\frac{z\left(\mathcal{R}_{q}^{\delta} f(z)\right)^{\prime}}{\mathcal{R}_{q}^{\delta} f(z)}}{b}-M\right|<M
$$

where $0<q<1, \delta>-1, M>\frac{1}{2}$, and $b \in \mathbb{C}^{*}=\mathbb{C} \backslash\{0\}$. It follows from [38] that $g \in$ $\mathcal{F}(1, m)=\lim _{q \rightarrow 1^{-}} \mathcal{F}_{0, q}(1, M)$ and only if for $z \in \mathbb{U}$

$$
\frac{z g^{\prime}(z)}{g(z)}=\frac{1+w(z)}{1-m w(z)}, \quad\left(m=1-\frac{1}{M}, M>\frac{1}{2}, w \in \Omega\right) .
$$

One can easily show that $f \in \mathcal{F}_{\lambda, q}(b, M)$ if and only if there is a function $g \in \mathcal{F}(1, M)$ such that

$$
\mathcal{R}_{q}^{\delta} f(z)=z\left(\frac{g(z)}{z}\right)^{b} .
$$

Thus from(1.16) and (1.17) it follows that $f \in \mathcal{F}_{\delta, q}(b, M)$ if and only if

$$
\frac{z\left(\mathcal{R}_{q}^{\delta} f(z)\right)^{\prime}}{\mathcal{R}_{q}^{\delta} f(z)}=\frac{1+[b(1+m)-m] w(z)}{1-m w(z)}, \quad(z \in \mathbb{U})
$$

where $0 \leq q<1, \delta>-1, M>\frac{1}{2}$, and $b \in \mathbb{C}^{*}$ and $w \in \Omega$.

By giving specific values of $\delta, q, b$ and $M$, we obtain the following important subclasses studied by various authors in the earlier works:

(1) $\lim _{q \rightarrow 1^{-}} \mathcal{F}_{\delta, q}(b, M)=\mathcal{F}_{\delta}(b, M)$ (Kumar et al. [26])

(2) $\lim _{q \rightarrow 1^{-}} \mathcal{F}_{0, q}(b, M)=\mathcal{F}(b, M)$ and $\mathcal{F}(b, \infty)=\mathcal{S}(b)$ (Nasr and Aouf [30] and [31])

(3) $\lim _{q \rightarrow 1^{-}} \mathcal{F}_{1, q}(b, M)=\mathcal{G}_{\lambda}(b, M)=\mathcal{G}(b, \infty)=\mathcal{C}(b)$ (Nasr and Aouf [29]) 
(4) $\lim _{q \rightarrow 1^{-}} \mathcal{F}_{0, q}\left(\cos \gamma e^{-i \gamma}, M\right)=\mathcal{F}_{\gamma, M}$ and $\lim _{q \rightarrow 1^{-}} \mathcal{F}_{1, q}\left(\cos \gamma e^{-i \gamma}, M\right)=\mathcal{G}_{\gamma, M}\left(|\gamma|<\frac{\pi}{2}\right)$ (Kulshrestha [25])

(5) $\lim _{q \rightarrow 1^{-}} \mathcal{F}_{0, q}(1, M)=\mathcal{F}(1, M)$ (Singh and Singh [39])

(6) $\mathcal{F}_{\delta, q}\left((1-\beta) \cos \alpha e^{-i \alpha}, M\right)=\mathcal{F}_{\delta, q}(\alpha, \beta, M) ;\left(0 \leq \beta<1,|\alpha|<\frac{\pi}{2}\right)$ if

$$
=\left\{f \in \mathcal{A}:\left|\frac{e^{i \alpha} \frac{z\left(\left(\mathcal{R}_{q}^{\delta} f(z)\right)^{\prime}\right.}{\mathcal{R}_{q}^{\delta} f(z)}-\beta \cos \alpha-i \sin \alpha}{(1-\beta) \cos \alpha}-M\right|<M, \quad z \in \mathbb{U}\right\},
$$

$\lim _{q \rightarrow 1^{-}} \mathcal{F}_{0, q}(\alpha, \beta, M)=\mathcal{F}(\alpha, \beta, M)$ and $\lim _{q \rightarrow 1^{-}} \mathcal{F}_{1, q}(\alpha, \beta, M)=\mathcal{G}(\alpha, \beta, M)$ (see Aouf[4, $5,8])$.

From the definitions of the classes $\mathcal{F}_{\delta, q}(b, M)$ and $\mathcal{F}(b, M)$ we observe that

$$
f \in \mathcal{F}_{\delta, q}(b, M) \Leftrightarrow \mathcal{R}_{q}^{\delta} f(z) \in \mathcal{F}(b, M) .
$$

Before we state and prove our main result we need the following definitions and lemmas.

Definition 1.1. [41] ( Subordinating Factor Sequence). A sequence $\left\{b_{n}\right\}_{n=1}^{\infty}$ of complex numbers is called a subordinating factor sequence if, whenever $f$ is analytic, univalent and convex in $\mathbb{U}$, we have the subordination given by

$$
\sum_{n=2}^{\infty} b_{n} a_{n} z^{n} \prec f(z) \quad\left(z \in \mathbb{U}, a_{1}=1\right) .
$$

Lemma 1.1. [41] The sequence $\left\{b_{n}\right\}_{n=1}^{\infty}$ is a subordinating factor sequence if and only if

$$
\Re\left(1+2 \sum_{n=1}^{\infty} b_{n} z^{n}\right)>0 \quad(z \in \mathbb{U}) .
$$

Now we prove the following lemma which gives a sufficient condition for function $f \in$ $\mathcal{F}_{\delta, q}(b, M)$

\section{Lemma 1.2. If}

$$
\sum_{n=2}^{\infty}[(n-1)+|b(1+m)+m(n-1)|] \Theta_{n}(q, \delta)\left|a_{n}\right| \leq|b(1+m)|
$$

then, $f \in \mathcal{F}_{\lambda, q}(b, M)$ where $0<q<1, \delta>-1, m=1-\frac{1}{M},\left(M>\frac{1}{2}\right)$ and $b \in \mathbb{C}^{*}$.

Proof. Suppose that the inequality (1.21) holds.Then for $z \in \mathbb{U}$, we have

$$
\left|z\left(\mathcal{R}_{q}^{\delta} f(z)\right)^{\prime}-\mathcal{R}_{q}^{\delta} f(z)\right|-\left|b(1+m) \mathcal{R}_{q}^{\delta} f(z)+m\left[z\left(\mathcal{R}_{q}^{\delta} f(z)\right)^{\prime}-\mathcal{R}_{q}^{\delta} f(z)\right]\right| .
$$

We have

$$
\left|\sum_{n=2}^{\infty}(n-1) \Theta_{n}(q, \delta) a_{n} z^{n}\right|
$$




$$
\begin{aligned}
& -\left|b(1+m)\left(z+\sum_{n=2}^{\infty} \Theta_{n}(q, \delta) a_{n} z^{n}\right)+m \sum_{n=2}^{\infty}(n-1) \Theta_{n}(q, \delta) a_{n} z^{n}\right| \\
\leq & \sum_{n=2}^{\infty}(n-1) \Theta_{n}(q, \delta)\left|a_{n}\right| r^{n} \\
& -\left\{|b(1+m)| r-\sum_{n=2}^{\infty}|b(1+m)|+m(n-1)\left|\Theta_{n}(q, \delta)\right| a_{n} \mid r^{n}\right\} \\
= & \sum_{n=2}^{\infty}[(n-1)+|b(1+m)+m(n-1)|] \Theta_{n}(q, \delta)\left|a_{n}\right| r^{n}-|b(1+m)| r .
\end{aligned}
$$

Letting $r \rightarrow 1^{-}$we have

$$
\begin{aligned}
& \mid z\left(\left(\mathcal{R}_{q}^{\delta} f(z)\right)^{\prime}-\mathcal{R}_{q}^{\delta} f(z)|-| b(1+m) \mathcal{R}_{q}^{\delta} f(z)+m\left[z\left(\mathcal{R}_{q}^{\delta} f(z)\right)^{\prime}-\mathcal{R}_{q}^{\delta} f(z)\right] \mid\right. \\
\leq & \sum_{n=2}^{\infty}[(n-1)+|b(1+m)+m(n-1)|] \Theta_{n}(q, \delta)\left|a_{n}\right|-|b(1+m)| \\
\leq & 0, \text { by (1.21). }
\end{aligned}
$$

Hence, it follows that

$$
\left|\frac{\frac{z\left(\mathcal{R}_{q}^{\delta} f(z)\right)^{\prime}}{\mathcal{R}_{q}^{\delta} f(z)}-1}{b(1+m)+m \frac{z\left(\mathcal{R}_{q}^{\delta} f(z)\right)^{\prime}}{\mathcal{R}_{q}^{\delta} f(z)}-1}\right|<1, z \in \mathbb{U} .
$$

Letting

$$
w(z)=\frac{\frac{z\left(\mathcal{R}_{q}^{\delta} f(z)\right)^{\prime}}{\mathcal{R}_{q}^{\delta} f(z)}-1}{b(1+m)+m \frac{z\left(\mathcal{R}_{q}^{\delta} f(z)\right)^{\prime}}{\mathcal{R}_{q}^{\delta} f(z)}-1},
$$

then $w(0)=0, w(z)$ is analytic in $\mathbb{U}$ and $|w(z)|<1$. Hence we have

$$
\frac{z\left(\mathcal{R}_{q}^{\delta} f(z)\right)^{\prime}}{\mathcal{R}_{q}^{\delta} f(z)}=\frac{1+[b(1+m)-m] w(z)}{1-m w(z)}, \quad(z \in \mathbb{U})
$$

which shows that $f \in \mathcal{F}_{\delta, q}(b, M)$.

Corollary 1.1. Let the function $f$ be defined by (1.1) be in the class $\mathcal{F}_{\delta, q}(b, M)$. Then

$$
\left|a_{n}\right| \leq \frac{|b(1+m)|}{[(n-1)+|b(1+m)+m(n-1)|] \Theta_{n}(q, \delta)}, \quad(n \geq 2) .
$$

The result is sharp for the function

$$
f_{n}(z)=z+\frac{|b(1+m)|}{[(n-1)+|b(1+m)+m(n-1)|] \Theta_{n}(q, \delta)} z^{n}, \quad(n \geq 2) .
$$


That is

$$
f_{2}(z)=z+\frac{|b(1+m)|}{[1+|b(1+m)+m|] \Theta_{2}(q, \delta)} z^{2}, \quad(n \geq 2) .
$$

Let $\mathcal{F}_{\delta, q}^{*}(b, M)$ denote the class of functions $f \in \mathcal{A}$ whose coefficients satisfy the condition (1.21). We note that $\mathcal{F}_{\delta, q}^{*}(b, M) \subseteq \mathcal{F}_{\delta, q}(b, M)$.

\section{Main Theorem}

Employing the techniques used earlier by Attiya [17],Frasin [20], Singh [38] Srivastava and Attiya [40] and others([7, 13, 14, 15, 20, 21], we obtain subordination relation involving the function classes $\mathcal{F}_{\delta, q}^{*}(b, M), \mathcal{F}_{\delta}^{*}(b, M), \mathcal{F}^{*}(b, M), \mathcal{G}^{*}(b, M)$ and $\mathcal{F}_{\delta, q}^{*}(\alpha, \beta, M)$.

Theorem 2.1. Let the function $f$ be defined by (1.1) be in the class $\mathcal{F}_{\delta, q}^{*}(b, M)$, where $0<q<1, \delta>$ $-1, M>\frac{1}{2}$, and $b \in \mathbb{C}^{*}$ with $\Re(b)>-\frac{m}{2(1+m)}(m>0)$ and $\Re(b)<-\frac{m}{2(1+m)}(m<0)$. Then

$$
\frac{[1+|b(1+m)+m|] \Theta_{2}(q, \delta)}{2\left[(1+|b(1+m)+m|) \Theta_{2}(q, \delta)+|b(1+m)|\right]}(f * g)(z) \prec g(z) \quad(z \in \mathbb{U} ; g \in \mathcal{K}),
$$

and

$$
\Re(f(z))>-\frac{[1+|b(1+m)+m|] \Theta_{2}(q, \delta)+|b(1+m)|}{[1+|b(1+m)+m|] \Theta_{2}(q, \delta)}, \quad(z \in \mathbb{U}) .
$$

The constant $\frac{[1+|b(1+m)+m|] \Theta_{2}(q, \delta)}{2\left[(1+|b(1+m)+m|) \Theta_{2}(q, \delta)+|b(1+m)|\right]}$ is the best estimate.

Proof. Let $\mathcal{F}_{\delta, q}^{*}(b, M)$ and let $g(z)=z+\sum_{n=2}^{\infty} c_{n} z^{n} \in \mathcal{K}$. Then

$$
\begin{aligned}
& \frac{[1+|b(1+m)+m|] \Theta_{2}(q, \delta)}{2\left[(1+|b(1+m)+m|) \Theta_{2}(q, \delta)+|b(1+m)|\right]}(f * g)(z) \\
= & \frac{[1+|b(1+m)+m|] \Theta_{2}(q, \delta)}{2\left[(1+|b(1+m)+m|) \Theta_{2}(q, \delta)+|b(1+m)|\right]}\left(z+\sum_{n=2}^{\infty} a_{n} c_{n} z^{n}\right) .
\end{aligned}
$$

Thus, by Definition 1.1 , the assertion of our theorem will hold if the sequence

$$
\left\{\frac{[1+|b(1+m)+m|] \Theta_{2}(q, \delta)}{2\left[(1+|b(1+m)+m|) \Theta_{2}(q, \delta)+|b(1+m)|\right]} a_{n}\right\}_{n=1}^{\infty}
$$

is a subordinating factor sequence, with $a_{1}=1$. In view of Lemma 1.1, this will be the case if and only if

$$
\Re\left(1+\sum_{n=1}^{\infty} \frac{[1+|b(1+m)+m|] \Theta_{2}(q, \delta)}{(1+|b(1+m)+m|) \Theta_{2}(q, \delta)+|b(1+m)|} a_{n} z^{n}\right)>0(z \in \mathbb{U}) .
$$


Now because $\left\{[(n-1)+|b(1+m)+m(n-1)|] \Theta_{n}(q, \delta)\right\}(n \geq 2, \delta>-1,0<q<1)$ is increasing function of $n(n \geq 2)$ we have

$$
\begin{aligned}
& \Re\left(1+\sum_{n=1}^{\infty} \frac{[1+|b(1+m)+m|] \Theta_{2}(q, \delta)}{(1+|b(1+m)+m|) \Theta_{2}(q, \delta)+|b(1+m)|} a_{n} z^{n}\right) \\
& =\Re\left(1+\frac{[1+|b(1+m)+m|] \Theta_{2}(q, \delta)}{(1+|b(1+m)+m|) \Theta_{2}(q, \delta)+|b(1+m)|} z\right. \\
& \left.+\frac{\sum_{n=2}^{\infty}[1+|b(1+m)+m|] \Theta_{2}(q, \delta) a_{n} z^{n}}{(1+|b(1+m)+m|) \Theta_{2}(q, \delta)+|b(1+m)|}\right) \\
& \geq\left(1-\frac{[1+|b(1+m)+m|] \Theta_{2}(q, \delta)}{(1+|b(1+m)+m|) \Theta_{2}(q, \delta)+|b(1+m)|} r\right. \\
& \left.-\frac{\sum_{n=1}^{\infty}[(n-1)+|b(1+m)+m(n-1)|] \Theta_{n}(q, \delta) a_{n} r^{n}}{(1+|b(1+m)+m|) \Theta_{2}(q, \delta)+|b(1+m)|}\right) \\
& \geq\left(1-\frac{[1+|b(1+m)+m|] \Theta_{2}(q, \delta)}{(1+|b(1+m)+m|) \Theta_{2}(q, \delta)+|b(1+m)|} r\right. \\
& \left.-\frac{|b(1+m)|]}{(1+|b(1+m)+m|) \Theta_{2}(q, \delta)+|b(1+m)|} r\right)=1-r>0 \quad(|z|=r) .
\end{aligned}
$$

Thus (2.5) holds true in $\mathbb{U}$. This proves the inequality (2.1). The inequality (2.2) follows by taking the convex function $g(z)=\frac{z}{1-z}=z+\sum_{n=2}^{\infty} z^{n}$ in (2.1). To prove the sharpness of the constant $\frac{[1+|b(1+m)+m|] \Theta_{2}(q, \delta)}{2\left[(1+|b(1+m)+m|) \Theta_{2}(q, \delta)+|b(1+m)|\right]}$ we consider the function $f_{0}$ given by

$$
f_{0}(z)=z-\frac{|b(1+m)|}{[1+|b(1+m)+m|] \Theta_{2}(q, \delta)+|b(1+m)|} z^{2},(z \in \mathbb{U})
$$

which is a member of the class $\mathcal{F}_{\delta, q}^{*}(b, M)$. Thus from $(2.1)$, we have

$$
\frac{[1+|b(1+m)+m|] \Theta_{2}(q, \delta)}{2\left[(1+|b(1+m)+m|) \Theta_{2}(q, \delta)+|b(1+m)|\right]} f_{0}(z) \prec \frac{z}{1-z} .
$$

It can easily verified that

$$
\min _{|z| \leq r}\left\{\Re\left(\frac{[1+|b(1+m)+m|] \Theta_{2}(q, \delta)}{2\left[(1+|b(1+m)+m|) \Theta_{2}(q, \delta)+|b(1+m)|\right]} f_{0}(z)\right)\right\}=-\frac{1}{2} \quad(z \in \mathbb{U}) .
$$

This shows that the constant $\frac{[1+|b(1+m)+m|] \Theta_{2}(q, \delta)}{2\left[(1+|b(1+m)+m|) \Theta_{2}(q, \delta)+|b(1+m)|\right]}$ cannot be replaced by a larger one, which completes the proof .

Letting $q \rightarrow 1^{-}$in Theorem 2.1, we obtain the following corollary. 
Corollary 2.1. Let the function $f$ be defined by (1.1) be in the class $\mathcal{F}_{\delta}^{*}(b, M)$, where $M>\frac{1}{2}$, and $b \in \mathbb{C}^{*}$ with $\Re(b)>-\frac{m}{2(1+m)}(m>0) ; \Re(b)<-\frac{m}{2(1+m)}(m<0)$ and satisfy the condition

$$
\sum_{n=2}^{\infty}[(n-1)+|b(1+m)+m(n-1)|] \Theta_{n}(1, \delta)\left|a_{n}\right| \leq|b(1+m)|
$$

where $\Theta_{2}(1, \delta)=\frac{\Gamma_{1}(2+\delta)}{[2-1]_{1} ! \Gamma_{1}(1+\delta)}=1+\delta$. Then

$$
\frac{[1+|b(1+m)+m|](1+\delta)}{2[(1+|b(1+m)+m 1|)(1+\delta)+|b(1+m)|]}(f * g)(z) \prec g(z) \quad(z \in \mathbb{U} ; g \in \mathcal{K}),
$$

and

$$
\Re(f(z))>-\frac{[1+|b(1+m)+m|](1+\delta)+|b(1+m)|}{[1+|b(1+m)+m|](1+\delta)}, \quad(z \in \mathbb{U}) .
$$

The constant $\frac{[1+|b(1+m)+m|](1+\delta)}{2[(1+|b(1+m)+m 1|)(1+\delta)+|b(1+m)|]}$ is the best estimate.

Letting $q \rightarrow 1^{-}$and taking $\delta=0$ in Theorem 2.1, we obtain the following corollary.

Corollary 2.2. Let the function $f$ be defined by (1.1) be in the class $\mathcal{F}^{*}(b, M)$, where $M>\frac{1}{2}$, and $b \in \mathbb{C}^{*}$ with $\Re(b)>-\frac{m}{2(1+m)}(m>0) ; \Re(b)<-\frac{m}{2(1+m)}(m<0)$ and satisfy the condition

$$
\sum_{n=2}^{\infty}[(n-1)+|b(1+m)+m(n-1)|]\left|a_{n}\right| \leq|b(1+m)| \text {. }
$$

Then

$$
\frac{[1+|b(1+m)+m|]}{(1+|b(1+m)+m 1|)+|b(1+m)|}(f * g)(z) \prec g(z) \quad(z \in \mathbb{U} ; g \in \mathcal{K}),
$$

and

$$
\Re(f(z))>-\frac{[1+|b(1+m)+m|]+|b(1+m)|}{1+|b(1+m)+m|}, \quad(z \in \mathbb{U}) .
$$

The constant $\frac{[1+|b(1+m)+m|]}{(1+|b(1+m)+m 1|)+|b(1+m)|}$ is the best estimate.

Letting $q \rightarrow 1^{-}$and taking $\delta=1$ in Theorem 2.1, we obtain the following corollary.

Corollary 2.3. Let the function $f$ be defined by (1.1) be in the class $\mathcal{G}^{*}(b, M)$, where $M>\frac{1}{2}$, and $b \in \mathbb{C}^{*}$ with $\Re(b)>-\frac{m}{2(1+m)}(m>0) ; \Re(b)<-\frac{m}{2(1+m)}(m<0)$ and satisfy the condition

$$
\sum_{n=2}^{\infty} n[(n-1)+|b(1+m)+m(n-1)|]\left|a_{n}\right| \leq|b(1+m)| .
$$

Then

$$
\frac{[1+|b(1+m)+m|]}{2[2(1+|b(1+m)+m 1|)+|b(1+m)|]}(f * g)(z) \prec g(z) \quad(z \in \mathbb{U} ; g \in \mathcal{K}),
$$

and 


$$
\Re(f(z))>-\frac{2[1+|b(1+m)+m|]+|b(1+m)|}{1+|b(1+m)+m|}, \quad(z \in \mathbb{U}) .
$$

The constant $\frac{[1+|b(1+m)+m|]}{2[2(1+|b(1+m)+m 1|)+|b(1+m)|]}$ is the best estimate.

If we put $b=(1-\beta) \cos \alpha e^{-i \alpha}\left(0 \leq \beta<1,|\alpha|<\frac{\pi}{2}\right)$, in Theorem 2.1, we obtain the next two result.

Corollary 2.4. Let the function $f$ be defined by (1.1) be in the class $\mathcal{F}_{\delta}^{*}(\alpha, \beta, M)$ and satisfy the condition

$$
\sum_{n=2}^{\infty}\left[(n-1)+\left|(1+m)(1-\beta) \cos \alpha e^{-i \alpha}+m(n-1)\right|\right] \Theta_{n}(q, \delta)\left|a_{n}\right| \leq|1+m|(1-\beta) \cos \alpha,
$$

where $0<q<1 ; M>\frac{1}{2}$, with $(1-\beta) \cos ^{2} \alpha>-\frac{m}{2(1+m)}(m>0)$ and $(1-\beta) \cos ^{2} \alpha<-\frac{m}{2(1+m)}(m<$ $0)$.Then

$$
\frac{\left[1+\left|(1+m)(1-\beta) \cos \alpha e^{-i \alpha}+m\right|\right] \Theta_{2}(q, \delta)}{2\left[\left(1+\left|(1+m)(1-\beta) \cos \alpha e^{-i \alpha}+m\right|\right) \Theta_{2}(q, \delta)+|1+m|(1-\beta) \cos \alpha\right]}(f * g)(z) \prec g(z)
$$

$(z \in \mathbb{U} ; g \in \mathcal{K})$ and

$$
\Re(f(z))>-\frac{\left[1+\left|(1+m)(1-\beta) \cos \alpha e^{-i \alpha}+m\right|\right] \Theta_{2}(q, \delta)+\left|(1+m)(1-\beta) \cos \alpha e^{-i \alpha}\right|}{\left[1+\left|(1+m)(1-\beta) \cos \alpha e^{-i \alpha}+m\right|\right] \Theta_{2}(q, \delta)},(z \in \mathbb{U}) .
$$

The constant $\frac{\left[1+\left|(1+m)(1-\beta) \cos \alpha e^{-i \alpha}+m\right|\right] \Theta_{2}(q, \delta)}{2\left[\left(1+\left|(1+m)(1-\beta) \cos \alpha e^{-i \alpha}+m\right|\right) \Theta_{q}(q, \delta)+|1+m|(1-\beta) \cos \alpha\right]}$ is the best estimate.

\section{Integral Means Inequalities}

Lemma 3.1. [27] If the functions $f$ and $g$ are analytic in $\Delta$ with $g \prec f$, then for $\eta>0$, and $0<r<1$,

$$
\int_{0}^{2 \pi}\left|g\left(r e^{i \theta}\right)\right|^{\eta} d \theta \leq \int_{0}^{2 \pi}\left|f\left(r e^{i \theta}\right)\right|^{\eta} d \theta
$$

In [35], Silverman found that the function $f_{2}(z)=z-\frac{z^{2}}{2}$ is often extremal over the family $\mathcal{T}$ denote the subset of $\mathcal{A}$ comprising of functions

$$
f(z)=z-\sum_{n=2}^{\infty}\left|a_{n}\right| z^{n} \quad(z \in \mathbb{U})
$$

and applied this function to resolve his integral means inequality, conjectured in [36] and settled in [37], that

$$
\int_{0}^{2 \pi}\left|f\left(r e^{i \theta}\right)\right|^{\eta} d \theta \leq \int_{0}^{2 \pi}\left|f_{2}\left(r e^{i \theta}\right)\right|^{\eta} d \theta
$$


for all $f \in \mathcal{T}, \eta>0$ and $0<r<1$. In [37], Silverman also proved his conjecture for the subclasses of starlike and convex functions of order $\alpha(0 \leq \alpha<1)$.

Applying Lemma 3.1 and Lemma 1.2 , we prove the following result.

Theorem 3.1. Suppose $f \in \mathcal{F}_{\delta, q}^{*}(b, M), \eta>0$, and $f_{2}(z)$ is defined by

$$
f_{2}(z)=z-\frac{|b(1+m)|}{[1+|b(1+m)+m|] \Theta_{2}(q, \delta)} z^{2}
$$

Then for $z=r e^{i \theta}, 0<r<1$, we have

$$
\int_{0}^{2 \pi}|f(z)|^{\eta} d \theta \leq \int_{0}^{2 \pi}\left|f_{2}(z)\right|^{\eta} d \theta
$$

Proof. For $f(z)=z-\sum_{n=2}^{\infty}\left|a_{n}\right| z^{n}$, (3.3) is equivalent to proving that

$$
\int_{0}^{2 \pi}\left|1-\sum_{n=2}^{\infty}\right| a_{n}\left|z^{n-1}\right|^{\eta} d \theta \leq \int_{0}^{2 \pi}\left|1-\frac{|b(1+m)|}{[1+|b(1+m)+m|] \Theta_{2}(q, \delta)} z\right|^{\eta} d \theta .
$$

By Lemma 3.1, it suffices to show that

$$
1-\sum_{n=2}^{\infty}\left|a_{n}\right| z^{n-1} \prec 1-\frac{|b(1+m)|}{[1+|b(1+m)+m|] \Theta_{2}(q, \delta)} z .
$$

Setting

$$
1-\sum_{n=2}^{\infty}\left|a_{n}\right| z^{n-1}=1-\frac{|b(1+m)| w(z)}{[(n-1)+|b(1+m)+m(n-1)|] \Theta_{n}(q, \delta)},
$$

and using (1.21), we obtain $w(z)$ is analytic in $\Delta, w(0)=0$, and

$$
\begin{aligned}
|w(z)| & =\left|\sum_{n=2}^{\infty} \frac{[1+|b(1+m)+m|] \Theta_{2}(q, \delta)}{|b(1+m)|}\right| a_{n}\left|z^{n-1}\right| \\
& \leq|z| \sum_{n=2}^{\infty} \frac{[(n-1)+|b(1+m)+m(n-1)|] \Theta_{n}(q, \delta)}{|b(1+m)|}\left|a_{n}\right| \\
& \leq|z| .
\end{aligned}
$$

This completes the proof of Theorem 3.1.

\section{Acknowledgments}

We authors record our sincere thanks to the referees for their valuable comments to revise the paper in present form. 


\section{References}

[1] H. Aldweby and M. Darus, Some subordination results on q-analogue of Ruscheweyh differential operator, Abst. Appl. Anal., 2014, (2014), Article ID 958563, 1-6.

[2] M. H. Annaby and Z.S.Mansour, q-Fractional Calculus and Equations, Lecture Notes in Maths, 2056, Springer-Verlag Berlin, Heidelberg 2012.

[3] M. K. Aouf, A. Shamandy, A. O. Mostafa and F. El-Emam, Subordination results associated with $\beta$-uniformly convex and starlike functions, Proc. Pakistan Acad. Sci. 46(2),(2009), 97-101.

[4] M. K. Aouf, Bounded p-valent Robertson functions of order $\alpha$, Indian J. Pure Appl. Math 16(7),(1985), 775-790.

[5] M. K. Aouf, Bounded spiral-like functions with fixed second coefficients InterJ.Math.Math. Sci. 12(1), (1989), 113-118.

[6] M. K. Aouf, On a new criteria for univalent functions of order $\alpha$, Rend.Math.Series-II, (1991), 47-59.

[7] M. K. Aouf, Subordination properties for a certain class of analytic functions defined by the Salagean operator, Appl. Math. Lett. 22(10),(2009), 1581-1585.

[8] M. K. Aouf, Bounded p-valent Robertson functions defined by using a differential operator, J. Frankl. Inst. 347(10),(2010), 1927-1941.

[9] M. K. Aouf and H.E. Darwish, On inequalities for certain analytic functions involving Ruscheweyh derivative, J. Math., 21(4),(1995), 387-393.

[10] M. K. Aouf, H.E. Darwish and A.A.Attiya, A remark on certain regular functions defined by Ruscheweyh derivative, Proc.Pakistan.Acad.Sci., 37(1)(2000),67-69.

[11] M. K. Aouf, and A.A.Al-Dohiman, Fixed second coefficient for certain subclasses of starlike functions with negative coefficients, Demonstratio Math., 38(3),(2005),551-565.

[12] M. K. Aouf and H.M.Hossen, Notes on certain classes of analytic function defined by Ruscheweyh derivative, Taiwense.T .Math., 1(1), (1997),11-19.

[13] M. K. Aouf and A. O. Mostafa, Some Subordination results for classes of analytic functions defined by the Al-Aboudi operator, Arch. Math., 92(2009), 279-286.

[14] M. K. Aouf, A. Shamandy, A. O. Mostafa and E. A. Adwan, Subordination results for certain class of analytic functions defined by convolution, Rend. del Circols Mat.di Palermo, no 60,(2011), 255-262.

[15] M. K. Aouf, A. Shamandy, A. O. Mostafa and E. A. Adwan, Subordination theorem of analytic functions defined by convolution, Complex Anal. Operator Theory, 7,(2013), 1117-1126.

[16] A. Aral, V. Gupta and R. P. Agarwal, Applications of q-Calculus in Operator Theory, Springer, New York, 2013.

[17] A.A. Attiya, On some application of a subordination theorems, J. Math. Anal. Appl. 311 (2005), 489-494.

[18] T. Bulboacã, Differential Subordinations and Superordinations, Recent Results, House of Scientific Book Publ., Cluj-Napoca, 2005.

[19] R.A. El-Ashwah, M. K. Aouf and A.A. Hassan, Subordination results for new subclasses of analytic univalent functions, Thai. J. Math., 15(1),(2017),113-140.

[20] B.A, Frasin, Subordination results for a class of analytic functions defined by a linear operator, J. Inequal. Pure Appl. Math. 7: (2006), 1-7.

[21] B. A. Frasin and G. Murugusundaramoorthy, A subordination results for a class of analytic functions defined by q-differential operator, Ann. Univ. Paedagog. Crac. Stud. Math. 19 (2020), 53-64.

[22] G. Gasper and M. Rahman, Basic Hypergeometric series, Cambridge Univ.Press, New York,1990.

[23] F. H. Jackson, On q-functions and a certain difference operator, Transactions of the Royal Society of Edinburgh, 46(1908), 253-281.

[24] S. Kanas and D. Răducanu, Some subclass of analytic functions related to conic domains, Math. Slovaca 64(2014), no. 5, 1183-1196.

[25] P. K. Kulshrestha, Bounded Robertson, Rend.Math., 6 (9) (1976), 137-150.

[26] V. Kumar, S.L.Shukla and A.M. Chaudhary, On a class of certain analytic functions of complex order, Tamkang J.Math.,21(2),(1990), 1-9.

[27] J. E. Littlewood, On inequalities in theory of functions, Proc. London Math. Soc. 23(1925), 481-519.

[28] S. S. Miller and P. T. Mocanu, Differential subordinations, Monographs and Textbooks in Pure and Applied Mathematics, 225, Dekker, New York, 2000. 
[29] M. A. Nasr and M. K. Aouf, On convex functions of complex order, Mansoura Bull. Sci., 8 (1982), 565-582.

[30] M. A. Nasr and M. K. Aouf, Bounded starlike functions of complex order, Proc. Indian Acad.Sci.,Math.,92 (1983), 97-102.

[31] M. A. Nasr and M. K. Aouf, Starlike function of complex order, J. Natur. Sci. Math., 25 (1985),1-12.

[32] S.Ruscheweyh, New criteria for univalent functions, Proc. Amer. Math. Soc. 49 (1975), 109-115.

[33] T. M. Seoudy and M. K. Aouf, Convolution properties for certain classes of analytic functions defined by q-derivative operator, Abstr. Appl. Anal., Vol. 2014, no. Article ID 846719, pp. 1-7, 2014, doi: 10.1155/2014/846719.

[34] T. M. Seoudy and M. K. Aouf, Coefficient estimates of new class of $q$-starlike and q-convex functions of complex order. J. Math. Inequal., 10,(2016), 135-145.

[35] H. Silverman. Univalent functions with negative coefficients, Proc. Amer. Math. Soc. 51(1975),109-116.

[36] H. Silverman, A survey with open problems on univalent functions whose coefficients are negative. Rocky Mt. J.Math. 21(1991),1099-1125.

[37] H. Silverman, Integral means for univalent functions with negative coefficients, Houston J. Math. 23(1997),169-174.

[38] S. Singh, A subordination theorems for spirallike functions, IJMMS, 24(7) (2000), 433-435.

[39] R. Singh and V.Singh, On a class of bounded starlike functions, Indian J. Pure Appl. Math.,5,(1974),733-740.

[40] H.M. Srivastava and A.A. Attiya, Some subordination results associated with certain subclasses of analytic functions, J. Inequal. Pure Appl. Math., 5(4) (2004), Article 82, 1-6.

[41] H.S. Wilf, Subordinating factor sequence for convex maps of the unit circle, Proc. Amer. Math. Soc. 12 (1961), 689-693. 